\title{
ESTUDO PRELIMINAR DO ELDER MEMORY TEST E RELAÇÃO COM NIVEL DE ESCOLARIDADE
}

\author{
José Maria Montiel \\ Daniel Bartholomeu² \\ Juliana F. Cecato ${ }^{3}$ \\ Luana Luz Bartholomeu \\ José Eduardo Martinelli5
}

resumo

Este estudo tem como objetivos desenvolver uma prova para avaliação dos componentes da memória, denominado Elder Memory Test, e posteriormente buscar evidências de validade

1 Graduado em Psicologia, Doutor em Psicologia pela Universidade São Francisco. Professor do Programa de Pós Graduação Strictu Sensu em Psicologia Educacional do Centro Universitário FIEO. E-mail: montieljm@hotmail.com.

2 Graduado em Psicologia, Doutor em Psicologia pela Universidade São Francisco. Professor do Programa de Pós Graduação Strictu Sensu em Psicologia Educacional do Centro Universitário FIEO. E-mail: daniel.bartholomeu@gmail.com.

3 Graduada em Biologia e Psicologia. Especialista em Psicopedagogia. Mestre em Ciências da Saúde pela Faculdade de Medicina de Jundiaí. Professora do curso de Pós Graduação Lato Sensu do UNISAL - Americana. E-mail: cecatojuliana@hotmail.com.

4 Graduada em Psicologia. Especialista em Psicopedagogia. Psicóloga Clínica. E-mail: Iuanasilvaluz@ gmail.com.

5 Graduado em Medicina. Doutor pela Unicamp. Professor do Programa de Pós Graduação Strictu Sensu em Ciências da Saúde da Faculdade de Medicina de Jundiaí. E-mail: drmartinelli@hotmail.com. 
para este instrumento de avaliação da memória, correlacionando o desempenho do teste de memória com as variáveis Escolaridade e Idade. Foram participantes uma amostra composta por 102 indivíduos com média de idade de 67 a 89, sendo que 73,5\% corresponde ao sexo feminino. Foi utilizado neste estudo um instrumento de avaliação para Memória, denominado Elder memory Test, que inclui os principais componentes de memória. Os resultados apontaram que os idosos apresentam diferentes médias em relação à escolaridade e idade, corroborando os estudos com esta população.

palavras-chave

Psicodiagnóstico. Avaliação Psicológica. Geriatria. Aprendizagem. Demência

\section{Introdução}

Segundo Neri (2007), o envelhecimento populacional tem sido um processo em pleno crescimento; com os avanços das ciências, gradativamente vem se mudando o estereótipo de incapacidade para uma visão mais otimista, considerando a velhice como um novo processo no qual oportunidades de melhores condições etárias tem sido propostos. Neste sentido, mudanças na visão social sobre o envelhecimento consequentemente altera a visão que o próprio idoso tem de si mesmo. Essa percepção subjetiva do envelhecimento contribui de uma maneira positiva tanto para a mudança do estereótipo social quanto para o que se esperar do futuro. Dados apontam que a população com 65 anos significará 7,7\% da população brasileira até 2020 (NERI, 2004). Segundo Ribeiro et al. (2007), a velhice é resultante de um processo de ganhos e perdas e são justamente o processo de perdas que caracterizam a velhice como um desafio social. Especialmente o declínio das capacidades cognitivas, as quais impossibilitam a tomada de decisão pelo idoso, deixando-o incapaz de realizar suas atividades do cotidiano e consequentemente interferindo na sua qualidade de vida (BATISTA; CRISPIM, 2012). Neste sentido, os desafios nesta compreensão estão relacionados ao papel da funcionalidade cognitiva, sendo este um tema bastante discutido nos estudos sobre o envelhecimento devido ao alto risco desta população desenvolver síndromes demenciais (RIBEIRO et al., 2007). 
Um fator comumente atrelado ao aumento da faixa etária e, consequentemente ao aumento da expectativa de vida, são as doenças neurodegenerativas como as demências do tipo Alzheimer, Vascular, Corpos de Lewy e Frontotemporais, que se tornam mais prevalentes na terceira idade e merecem maior atenção (CARAMELLI et al., 2002; TEIXEIRA et al., 2008). Por exemplo, estudos apontam que a doença de Alzheimer (DA) representa de 50 a $70 \%$ dos casos de demência em população acima dos 65 anos, aumentando a prevalência com o aumento da faixa etária (TEIXEIRA et al., 2008). Com tudo, os procedimentos de investigação diagnóstica das síndromes demenciais se baseiam em uma série de exames, entre os quais se podem citar os exames clínicos, neuropsicológicos, laboratoriais e de neuroimagem para se estabelecer o diagnóstico diferencial (CARAMELLI et al., 2002; GOTTLIEB et al., 2011).

Em relação às alterações observadas especificamente nos casos de idosos com demências do tipo Alzheimer o processo neurodegenerativo se inicia com a deterioração da região cerebral denominada hipocampo, com posterior comprometimento de regiões corticais (CARAMELLI et al., 2002; TEIXEIRA et al., 2008). Essas alterações provocam um quadro clínico característico como perda de memória episódica e alterações comportamentais de uma maneira gradual. Isso significa dizer que a memória para fatos recentes é o fator cognitivo mais comprometido, porém a memória para fatos antigos ainda está preservada. Segundo estudos, por exemplo Charchat-Fichman et al. (2005), o paciente com demências do tipo Alzheimer apresenta dificuldade em armazenar e evocar informações que foram aprendidas há poucos minutos, mas fatos que aconteceram em sua infância podem ser lembrados com detalhes. Devido a tais alterações as atividades básicas e instrumentais da vida diária também se tornam comprometidas a medida que a doença progride.

Um dos aspectos a serem considerados é a autonomia do idoso, fundamental quanto à capacidade de escolha e de liberdade para tomar decisões (GOTTLIEB et al., 2011; LACERDA et al., 2013; NERI, 2010; FORMIGA, et al., 2000; SANTOS; PAVARINI, 2011; TORRES et al., 2009). Outro ponto a ser considerado na capacidade funcional diz respeito às habilidades cognitivas do idoso. O declínio da cognição, observado por meio de testes psicológicos, impossibilita o indivíduo de tomar decisões, contribuindo na dependência em realizar atividades diárias e interfere na sua autonomia e qualidade de vida (NERI, 2010; RIBEIRO et al., 2007; SCHMIDT et al., 2009).

Diante do arcabouço teórico apresentado atualmente na literatura, podem ser observados, entre os métodos de avaliação dos declínios cognitivos, os procedimentos de avaliação com caráteres neuropsicológicos cujo quais têm 
apresentado significativo valor preditivo especialmente em relação ao que concerne ao desempenho e habilidades do individuo (CECATO et al., 2012; MONTIEL et al., 2013). Neste sentido, especificamente por serem caracterizadas como avaliações específicas, como no caso de avaliação de componentes de memória, de funções executivas, entre outras habilidades e/ou funções cognitivas (SANTOS; PAVARINI, 2011; MARTINELLI et al., 2014).

Dentre os métodos de avaliação de tais quadros demenciais os procedimentos de investigação neuropsicológicos tem tido significativa pertinência, especialmente na relação entre desempenho e estrutura neurológica (ARGIMON et al., 2005). Neste sentido, segundo Agrell et al. (1998), Heink et al. (2003), e Nitrini et al. (2005), por apresentar avaliações mais complexas e específicas, a avaliação neuropsicológica tem apresentado êxito em diferentes avaliações, tais como, memória recente, funções executivas, a linguagem, a compreensão, a praxia, dentre outras funções. Assim, a neuropsicologia pode ser considerada como ciência que buscar relacionar a atividade do sistema nervoso com o funcionamento psicológico, tanto em condições normais quanto patológicas, incluindo o estudo do desenvolvimento e dos distúrbios cognitivos, emocionais e de personalidade. A Avaliação Neuropsicológica envolve o estudo intensivo do comportamento por meio de entrevistas, questionários e testes normatizados que permitam obter desempenhos relativamente precisos (MONTIEL, 2005). Tais estudos preconizam que o dano cerebral observado é considerado um fenômeno multidimensional mensurável e que requer uma abordagem de avaliação multidimensional.

Em relação aos procedimentos de avaliação neuropsicológica Nitrini et al. (2005) aponta complicadores neste tipo de avaliação, dentre estes, o uso de testes padronizados e validados em outros países e 'trazidos' para serem utilizados na população brasileira. Nesse contexto, as discussões na área das avaliações psicológicas e neuropsicológicas se tornam fundamentais a fim de buscar evidências de que tais instrumentos avaliem o que estão se dispondo a avaliar, ou seja, avalie o comportamento-alvo. Para que o teste seja capaz de avaliar aquilo que foi proposto, é necessário obter evidências de validade para determinadas populações. A validade de um teste deve levar em consideração o contexto que os indivíduos estão inseridos, ou seja, o teste será útil se aplicado dentro de uma determinada população levando em consideração diversas variáveis dessa população (URBINA, 2007; CAMPOS, 2008). Neste sentido, a avaliação deve gerar explicação de modelos do desempenho cognitivo, intacto e alterado, em termos de prejuízos de um ou mais dos componentes do modelo de funcionamento cognitivo, e derivar conclusões sobre os processos cognitivos, intactos, a partir de modelos de capacidades deficitárias e intactas vistos. 
Mensurar as capacidades cognitivas dos idosos, mesmo naqueles que apresentam comprometimento mental, é importante para os profissionais envolvidos com tais pacientes, especificamente no que diz respeito a programas de reabilitação e estimulação cognitiva (YASSUDA et al., 2006; MONTIEL et al., 2013). Estudos como o de Cooper et al. (2001; 2004) observaram melhoras em pacientes idosos após estimulação cognitiva indicada depois da avaliação neuropsicológica. Acrescentam que quando comparados os testes psicométricos em idosos com diferentes estágios de demência e com um grupo controle num intervalo de uma semana, os resultados não demonstraram piora no desempenho dos pacientes com demência, porém apontaram que idosos normais melhoraram significativamente nas avaliações após procedimentos de estimulação.

Em relação aos componentes e aos declínios cognitivos avaliados na demência do tipo Alzheimer diversos aspectos são considerados, entre estes a memória tem sido foco de atenção em diversos estudos, pois apresenta significativa importância nestes quadros. Diversas são as definições deste constructo, a mais comumente utilizada é que a memória pode ser definida como a capacidade de aquisição, armazenamento e evocação da informação registrada (CORRÊA, 2008; MONTIEL et al., 2013). Estudos como os de Yassuda (2002), Corrêa (2008) e Nitrini (2005) apontam que a memória semântica, implícita, de procedimento e de longo prazo são mais resistentes nestes quadros, e não são tão afetadas durante o processo de envelhecimento quando comparadas às memórias episódica, explícita e de curto prazo.

Nos apontamentos descritos por Nitrini (2005), pode ser observado que a dificuldade de se evocar novas informações é um dos primeiros sinais que se observa na demência do tipo Alzheimer, comumente se pode observar com maior prevalência déficit na memória de curto prazo, sendo esta dividida em primária (manutenção passiva de informações novas) e em memória operacional, também chamada de working memory (componente ativo da memória de curto prazo e responsável por manter as informações novas). Ainda segundo o mesmo autor, a memória operacional apresenta um componente responsável pela ligação entre os sistemas subordinados e a memória de longo prazo, denominado de executivo central, sendo esta uma função exercida pelo lobo frontal e que, entre outras funções, supervisiona informações que serão codificadas, armazenadas e evocadas concomitantemente a sua entrada no sistema, que segundo Yassuda (2002) e Corrêa (2008) é uma das primeiras funções comprometidas nos déficits cognitivos provocados pela demência do tipo Alzheimer. Em outro estudo, Charchat-Fichman et al. (2001) estudaram testes informatizados que avaliavam a memória em 40 pacientes 
com diagnóstico de DA e compararam esse grupo com 73 idosos normais. Os resultados demonstraram que há o prejuízo da memória no que se refere a acontecimentos recentes como os primeiros sintomas da demência do tipo Alzheimer, seguidos do declínio da memória semântica quando comparados aos participantes sem sintomas.

Em outros apontamentos Silva et al. (2002) e Martinelli et al. (2014) descrevem que os sintomas como esquecimentos ou comprometimento da memória em pessoas idosas são considerados pelos familiares de pacientes como um processo natural do envelhecimento e por essa razão há demora na procura por uma investigação clínica e neuropsicológica efetiva dessas alterações.Outras considerações de Abreu et al. (2005) apontam que, por se tratar de uma doença que gera altos custos para a família do portador e para a sociedade, existe pouca preocupação do sistema de saúde em se fazer diagnósticos precoces, os quais permitiriam intervenções terapêuticas que ajudassem a diminuir os comprometimentos e o estresse de seus cuidadores e que propiciaria uma melhora na qualidade de vida do portador, como por exemplo, a manutenção da autonomia desses pacientes (CHARCHAT-FICHMAN et al., 2005). Bennett et al. (2000) acrescentam que a piora dos idosos em tarefas que avaliam a aprendizagem pode ser considerada um fator preditivo para o diagnóstico de demência, pois os comprometimentos ocasionados pelo executivo central, observado nas tarefas de aprendizagem, poderiam ocasionar dificuldades de autonomia cognitiva, o que demonstraria uma síndrome demencial em evolução.

É reconhecido que, em decorrência dos apontamentos anteriormente descritos, os quadros demências acabam sendo hipervalorizados ou descontextualizados. Assim, tal fato impulsiona profissionais a buscarem soluções inteligentes, integradas e efetivas para resolução ou amenização destes problemas. É também sabido que, no Brasil, profissionais que trabalham com populações demenciais se deparam com um problema bastante recorrente e grave, que é a falta de ferramentas específicas para avaliação e intervenção; neste sentido, parte do problema é decorrente da própria falta de instrumentos, e/ou pela escassez de instrumentos validos e normatizados disponíveis para pesquisa e diagnóstico com esta população. Desta forma, é de suma relevância estudos que visem minimizar tal carência. Assim, este estudo tem como objetivos desenvolver uma prova para avaliação dos componentes da memória, denominado Elder Memory Test, e posteriormente buscar evidencias de validade para este instrumento de avaliação da memória, correlacionando o desempenho do teste de memória com as variáveis Escolaridade e Idade. 


\subsection{Participantes}

Este estudo, de caráter transversal, foi realizado com 102 idosos, com idade igual ou superior a 60 anos, de ambos os sexos, que frequentam um grupo aberto à terceira Idade. Os idosos participaram da pesquisa por livre e espontânea vontade, conforme a assinatura dos termos de consentimento. Os resultados em relação à população estudada encontram-se descritos no item Resultados.

\subsection{Instrumentos}

Para realização deste estudo foi desenvolvido um instrumento de avaliação da Memória para idoso, aqui denominado Elder memory Test, que incluísse os principais componentes de memória segundo o arrazoado teórico pesquisado, ou seja, memória imediata, de evocação e tardia; memória de reconhecimento; memória operacional e componentes de memória tardia, conforme descritos abaixo.

\subsection{Avaliação de Memória Imediata, de Evocação e Tardia}

Na avaliação deste componente, a avaliação da memória foi realizada por meio de slides realizados no programa Power Point (versão Windows 2003) em que 20 figuras foram apresentas com um tempo de memorização de 2 minutos. Logo após a exibição das figuras, pediu-se para que os participantes escrevessem na folha de respostas as figuras quais se lembravam de ter sido apresentadas. Em seguida, foram apresentadas novamente para a memorização as 20 figuras, com um tempo de 2 minutos. Posteriormente, foi apresentado um distrator de 1 minuto e 30 segundos e, após a realização da tarefa, foi pedido para os participantes evocarem o que se lembravam das 20 figuras. Após, as figuras foram exibidas, com um tempo de 2 minutos para a memorização. Os distratores foram apresentados em seguida, agora se aumentando o tempo, ou seja, distratores de 2 e 3 minutos após a exibição das 20 figuras. Depois do último distrator de 3 minutos, foram exibidas as 20 figuras novamente para a memorização, também com um tempo de 2 minutos. A evocação foi realizada desta vez após um tempo mínimo de 15 minutos, a fim de avaliar a memória tardia. 
A avaliação da memória de reconhecimento foi realizada por meio da criação de uma figura utilizando os programas Adobe Photoshop 7.0.1 e Macromidia Fireworks 6.0 for Windows, com 23 estímulos. Essa figura foi apresentada por 3 minutos. Em seguida, foi pedido ao participante que circulasse na folha de respostas os estímulos apresentados na figura anterior, sendo que nessa folha estavam presentes 19 estímulos que não foram mostrados na figura. Os participantes tiveram até 2 minutos para realização da tarefa.

\subsection{Avaliação de Memória Operacional}

Para avaliação da memória operacional, foi solicitado para os participantes que memorizassem três figuras que não se correlacionam, durante 1 minuto e 30 segundo. Em seguida, foi solicitado que o participante escreva o nome das figuras apresentadas por ordem alfabética de acordo com a primeira letra de cada palavra. Os participantes tiveram 1 minuto e 30 segundos para escrever o nome das figuras. O teste de memória operacional apresentava três subtestes, começando com três estímulos e terminando com cinco. Os participantes receberam 1 ponto para cada memorização correta e 1 ponto para cada palavra escrita na ordem certa. Se o participante não escrevesse a palavra na ordem alfabética, só receberia pontuação para a memorização da palavra, mas perderia 1 ponto por escrever a palavra na ordem errada.

\subsection{Avaliação de Memória Tardia}

Para esta avaliação foi solicitado aos participantes que evocassem os 20 estímulos que foram exibidos no primeiro slide. Seriam pontuados os acertos em cada uma das situações estudadas tanto para memória de evocação, tardia, de reconhecimento e operacional, atribuindo ponto para cada um desses e zero para erros. A soma em cada tarefa forneceria a pontuação total das pessoas.

\subsection{Procedimento}

Após aprovação pelo comitê de Ética em Pesquisa da Faculdade de Medicina de Jundia de acordo com o protocolo numero 54/2011 e colhida as autorizações por meio dos termos de consentimentos livre e esclarecidos, a aplicação dos instrumentos iniciou-se. Após, objetivou-se fazer a validação 
do teste (Elder memory Test), por meio da técnica de validade por critério, que segundo os apontamentos de Campos (2008) a validade por critério é utilizada quando se pretende medir uma segunda variável que está relacionada com aquela característica que se pretende avaliar. Tendo tais pressupostos os escores do teste de memória foram comparados com as variáveis Idade e Escolaridade da amostra avaliada. Em relação a análise dos dados coletados, esta foi feita através do programa SPSS, onde foram realizadas estatísticas descritivas quanto a distribuição da amostra (idade, gênero, escolaridade). As diferenças entre os grupos de idade (60 a 69 anos; 70 a 79 anos; > 80 anos) e escolaridade ( 1 a 4 anos; 5 a 8 anos; 9 a 11 anos; > 11 anos) foram feitas por meio da análise de Kruskal-Wallis. Entre o grupo de idade de 60 a 69 anos e $>80$ anos, foi utilizado o teste de Mann-Whitney. Coeficientes de correlação de Pearson foram realizados entre as variáveis Idade e Escolaridade com os escores dos testes de memória. A análise de consistência interna de cada prova do Elder memory Test foi realizada pela prova de Kuder Richardson.

\section{Resultados}

Em relação aos resultados, este estudo contou com uma amostra composta por 102 participantes com média de idade de 67,89 $($ Mediana = 67; mínimo = 60 ; máximo = 90, desvio padrão $[\mathrm{dp}]=6,32$ ) e com $73,5 \%$ corresponde ao sexo feminino. A figura 1 apresenta a frequência e porcentagem dos níveis de escolaridade dos participantes, sendo que a grande maioria dos participantes apresentou alta escolaridade, ou seja, $56,9 \%$ da amostra tinham mais do que nove anos de estudo.

Figura 1 - Freqüência e porcentagem dos níveis de escolaridade dos participantes.

\section{Escolaridade}

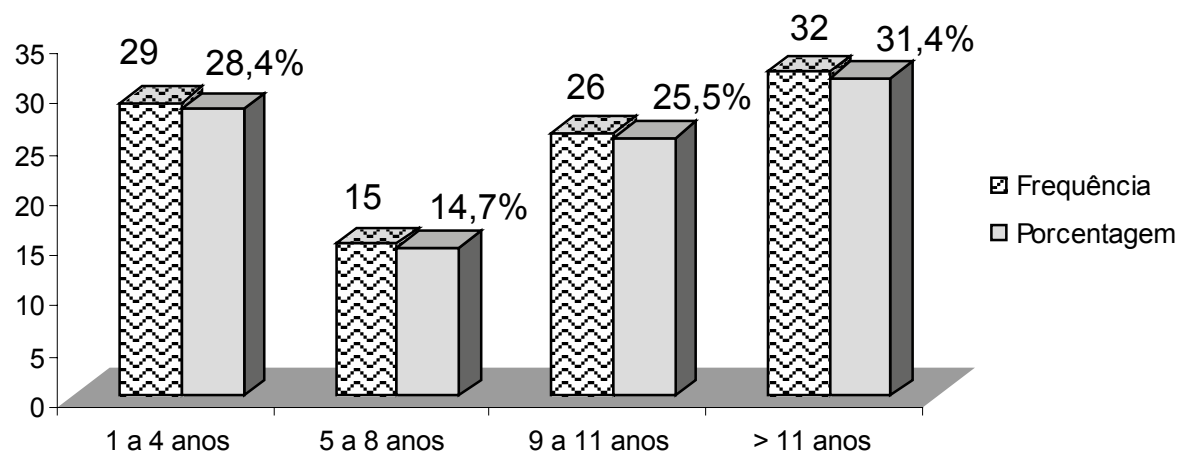


Quanto aos resultados com o teste de correlação, os dados demonstraram que a variável Escolaridade apresenta uma correlação fraca com os testes de memória: evocação $1(\mathrm{r}=0,18 ; \mathrm{p}=0,072)$, evocação $2(\mathrm{r}=0,27 ; \mathrm{p}=0,006)$, evocação 3 $(\mathrm{r}=0,25 ; \mathrm{p}=0,012)$, evocação $4(\mathrm{r}=0,23 ; \mathrm{p}=0,017)$, memória operacional 1 $(\mathrm{r}=0,25 ; \mathrm{p}=0,010)$, memória operacional $2(\mathrm{r}=0,29 ; \mathrm{p}=0,003)$, memória operacional $3(\mathrm{r}=0,28 ; \mathrm{p}=0,004)$, memória tardia $(\mathrm{r}=0,20 ; \mathrm{p}=0,048)$ e memória de reconhecimento $(\mathrm{r}=0,30 ; \mathrm{p}=0,003)$. Já no que tange à relação com a variável idade, foram encontradas coeficientes de correlação fraca e não significativas. As análises que mostraram diferenças significativas foram apenas nos testes de evocação $3(\mathrm{r}=0,31 ; \mathrm{p}=0,001)$ e $4(\mathrm{r}=0,34 ; \mathrm{p}<0,000)$, e na memória tardia $(r=0,40 ; p<0,000)$ com correlações negativas.

Em um segundo momento a análise de Mann-Whitney foi realizada com os grupos de idade entre 60 a 69 anos e 70 a 72 anos, sendo que os resultados não demonstraram diferenças significativas entres os testes de memória, com exceção do teste de evocação $3(p=0,016)$, evocação $4(p=0,041)$ e memória tardia $(p=0,014)$. É importante ressaltar que observam-se diferenças estatisticamente significativas entre os grupos de 60 a 69 anos e acima de 80 anos. Pode ser observado na tabela 1 que o grupo com mais anos de vida apresenta médias inferiores nos testes cognitivos quando comparados ao grupo de 60 a 69 anos.

Tabela 1 - Médias nos testes de memória e os grupos de entre 60 a 69 anos e acima de 80 anos.

\begin{tabular}{l|c|c|c}
\hline \multicolumn{1}{c|}{ Testes } & $\begin{array}{c}\text { 60 a 69 anos } \\
\text { Média }\end{array}$ & $\begin{array}{c}>80 \text { anos } \\
\text { Média }\end{array}$ & * \\
\hline Evocação1 & 11,35 & 6,6 & 0,003 \\
\hline Evocação 2 & 12,08 & 6,4 & 0,010 \\
\hline Evocação 3 & 15 & 9,4 & 0,009 \\
\hline Evocação 4 & 16,24 & 10,4 & 0,004 \\
\hline Reconhecimento & 11,95 & 10,4 & 0,349 \\
\hline Memória Operacional 1 & 2,87 & 2,4 & 0,626 \\
\hline Memória Operacional 2 & 3,43 & 2,8 & 0,226 \\
\hline Memória Operacional 3 & 3,98 & 2,8 & 0,130 \\
\hline Tardia & 17,16 & 9 & 0,001 \\
\hline
\end{tabular}


Em um terceiro momento de análises, separando os grupos pelo nível de escolaridade, encontrou-se diferenças estatisticamente significativas entre os quatro grupos de escolaridade e os testes de memória de evocação 2 $(\mathrm{p}=0,008)$, evocação $3(\mathrm{p}=0,015)$, evocação $4(\mathrm{p}=0,011)$, reconhecimento $(p=0,003)$, memória operacional $1(p=0,042), 2(p=0,018)$ e $3(p=0,007)$, como pode ser observado na tabela 2 , na comparação entre as médias dos testes e os grupos de idade.

Tabela 2 - Médias nos testes de memória (Elder memory Test) e os grupos entre 60 a 69 anos e acima de 80 anos.

\begin{tabular}{|c|c|c|c|c|c|}
\hline Testes & $\begin{array}{c}1 \text { a } 4 \text { anos } \\
\text { Média }\end{array}$ & $\begin{array}{l}5 \text { a } 8 \text { anos } \\
\text { Média }\end{array}$ & $\begin{array}{c}9 \text { a } 11 \text { anos } \\
\text { Média }\end{array}$ & $\begin{array}{l}>8 \text { anos } \\
\text { Média }\end{array}$ & ${ }^{*} p$ \\
\hline Evocação 1 & 10,00 & 11,20 & 11,27 & 11,50 & 0,102 \\
\hline Evocação 2 & 10,21 & 10,80 & 12,46 & 12,78 & 0,008 \\
\hline Evocação 3 & 12,90 & 13,47 & 15,38 & 14,87 & 0,015 \\
\hline Evocação 4 & 14,17 & 14,87 & 16,31 & 16,18 & 0,011 \\
\hline Reconhecimento & 10,13 & 12,40 & 11,23 & 13,50 & 0,003 \\
\hline Memória Operacional 1 & 2,59 & 2,87 & 3,0 & 2,9 & 0,042 \\
\hline Memória Operacional 2 & 2,59 & 3,53 & 3,19 & 3,69 & 0,018 \\
\hline Memória Operacional 3 & 2,96 & 3,67 & 4,08 & 4,25 & 0,007 \\
\hline Tardia & 14,69 & 16,47 & 17,27 & 16,66 & 0,095 \\
\hline
\end{tabular}

Foi realizada análise de consistência interna de cada uma das avaliações do Elder memory Test, pela prova de Kuder Richardson; esta prova é própria para itens com aspectos dicotômicos. Por meio das analises observou-se que os coeficientes oscilaram de 0,53 a 0,89. Desta maneira, pode-se afirmar que quanto mais próximo de 1 melhor nessa análise. Outro dado relevante é que somente a medida da evocação 1 apresentou KR abaixo do patamar exigido pelo conselho federal de Psicologia como mínimo aceitável para um teste de 0,60 . Consecutivamente, todas estiveram entre 0,73 a 0,89 . Na escala Total 1 , a exclusão de nenhum item melhoraria significativamente, sendo que o maior aumento seria para um coeficiente de 0,55 . Isso sugere que os itens nessa escala não estão altamente correlacionados (correlação ponto-bisserial) e que devem ser revistos. 
É importante ressaltar que diversos estudos apontam a importância dos testes neuropsicológicos para o diagnóstico diferencial da doença de Alzheimer, principalmente aqueles que avaliam a aprendizagem e memória viso-espacial (GAINOTTI et al., 1994; GIOVANNETTI et al., 2001; GOLD et al., 2007), os quais demonstram a importância na averiguação do funcionamento neuropsicológico. Cabe ainda apontar, por meio do arrazoado teórico analisado, que estudos realizados na população brasileira evidenciam, em sua maioria, que a avaliação neuropsicológica mais utilizada na investigação das síndromes demenciais nos idosos é o Cambrigde Cognitive Examination (CAMCOG), pois tem apresentando alta sensibilidade (100\%) e especificidade (95\%) para esta população (APRAHAMIAN et al., 2001; NUNES et al., 2008; LACERDA et al., 2013; SANTOS; PAVARINI, 2011). De acordo com os apontamentos de Aprahamian et al. (2011), em uma versão reduzida, o CAMCOG apresentou alta acurácia em diferenciar quadros de declínio cognitivo leve da doença de Alzheimer.

Seguindo ainda o anteriormente descrito, tendo por base o levantamento bibliográfico realizado para este estudo, se pode observar e evidenciar que os estudos e artigos publicados na área buscam deparar com um teste com maior valor preditivo para o diagnóstico diferencial das demências (APRAHAMIAN et al., 2001; NUNES et al., 2008; VALLS-PEDRET et al., 2011; CECATO et al., 2012; MONTIEL et al., 2013; MARTINELLI et al., 2014). Neste instrumento de avaliação, foco deste estudo, faltam dados consistentes sobre o seu valor preditivo em casos de comprometimento cognitivo, o que poderia ser resolvido a partir de aplicações juntamente com outros testes validados no Brasil, para efeito comparativo. Porém, como se trata de um estudo preliminar, os dados aqui demonstrados suprem este primeiro momento e abrem vias para novas avaliações neste contexto.

Neste estudo pode ser observado que os idosos apresentam diferentes médias em relação à escolaridade e idade, tais dados corroboram os achados do estudo realizado por Diniz et al. (2007), que verificaram a influência dessas variáveis no teste cognitivo Mini-exame do Estado Mental (MEEM) em 176 idosos. As análises mostraram diferenças estatisticamente significativas na pontuação do MEEM entre os diferentes níveis de escolaridade e idade. No estudo de Matallana et al. (2011) foi verificado também o desempenho de idosos no MEEM ao estudarem 2.861 participantes provenientes de populações hispânicas e encontrarem pontuações elevadas nos testes entre idosos com alta escolaridade. Ainda neste sentido, outro estudo que autentica os achados 
da presente pesquisa foi realizado por Brito-Marques et al. (2005), onde se avaliaram 253 participantes por meio do MEEM, e separou-se em grupos de idade igual a 60-65, 66-70, 71-75, 76-80, 81-85 e 86-90 anos, e a escolaridade variando de 1 a 4 anos, 5 a 8 anos e mais de 8 anos de estudo; os resultados apontaram diferenças estatisticamente significativas em relação as variáveis idade e escolaridade.

A partir do arrazoado teórico, e dos resultados anteriormente demonstrados neste estudo, se pode afirmar que a idade e a escolaridade demonstram ser variáveis importantes e que devem ser consideradas durante a entrevista e a anamnese neuopsicológica, especialmente em pessoas idosas. Tal afirmativa pode ser observada nos apontamentos realizados por Valls-Pedret et al. (2011), cujo estudo aplicou um teste de figuras em pacientes com declínio cognitivo e em idosos sem comprometimento - ou seja, 137 pessoas sem comprometimentos e 126 com sintomas para DA - cuja avaliação focava a memória episódica visual. O estudo apontou que o grupo mais jovem, com idade entre 50 a 59 anos, obteve médias superiores no teste quando comparado aos escores de pessoas com mais de 80 anos. É importante ressaltar que neste estudo o interessante foi que a diferença significativa foi observada no grupo controle, ou seja, foram encontradas diferenças de pontuações no teste mesmo entre os idosos que não apresentavam nenhuma evidência de síndrome demencial, o que reafirma a influência da variável idade no teste de memória (VALLS-PEDRET et al., 2011).

Pesquisadores como, por exemplo, Brito-Marques et al. (2005), Diniz et al. (2007), Matallana et al. (2011), Valls-Pedretet al. (2011), Cecato et al. (2012) e Montiel et al. (2013), corroboram com os resultados encontrados neste estudo, ou seja, idosos com mais anos de escolaridade obtêm maiores médias nos testes de memória quando comparados a idosos com menos anos de estudo. Neste mesmo sentido, o mesmo se aplica com o fator Idade, onde idosos mais jovens apresentam maiores médias nos testes quando comparados a idosos mais velhos. Em seus apontamentos, Diniz et al. (2007) referem-se como a alta escolaridade um fator protetor para demência, ou seja, os anos de estimulação cerebral provocada pelos estudos produz uma reserva cognitiva, o que faz diminuir o risco para se desenvolver quadros demenciais. Tal hipótese é aventada e reforçada por Yassuda et al. (2006), anteriormente, ao estudar os efeitos de treinos de memória em idosos. Em síntese, se pode afirmar que a idade e a escolaridade são variáveis consideradas como fatores de risco para síndrome demencial, ou seja, quanto maior a idade e menor a escolaridade do indivíduo mais chances da pessoa desenvolver declínio das funções cognitivas como apontados por Moraes et al. (2010) e Unverzaqt et al. (2011). 
Atualmente, no Brasil, diversos estudos têm enfocado os efeitos das variáveis sociodemográficas na saúde física e no desempenho cognitivo do idoso, sendo o MEEM o teste de rastreio mais utilizado, conforme evidenciado em estudos anteriormente descritos. Por exemplo, o estudo de Moraes et al. (2010) comprova os efeitos de variáveis como sintomas depressivos, idade e escolaridade no desempenho cognitivo dos idosos, sugerindo uma maior atenção aos profissionais de saúde na avaliação global de pessoas idosas (CECATO et al., 2012). Na presente pesquisa, torna-se importante ressaltar que faltou a coleta de dados com relação aos sintomas depressivos e outros mentais dos participantes, uma vez que é sabido que tais manifestações poderiam ser um aspecto que influenciasse negativamente no desempenho e execução deste estudo. Desta maneira, mesmo com ausências de alguns dados sintomatológicos e, apesar da falta de dados consistentes sobre a sensibilidade e especificidade em predizer quadros demenciais, pode-se concluir que o teste em questão Elder Memory Test, pode contribuir com a investigação diagnóstica de declínio cognitivo e pode, ainda, servir de futuras pesquisas de validade, sendo mais um instrumento diagnóstico, o qual poderá ser útil até mesmo com propósito de intervenções mais apuradas e específicas.

PRELIMINARY STUDY OF THE ELDER MEMORY TEST AND RELATIONS WITH ACADEMIC LEVEL

abstract

This study aims to develop a test to evaluate memory components (Elder Memory Test), and then look for validity evidence in assessing memory by correlating the memory test scores with age and educational level. One hundred and two subjects with mean age of 67 years and $73.5 \%$ female were studied. We utilized in this study an assessment instrument for memory, called memory Elder Test that includes the main components of memory. The results indicated that the elderly have different mean scores by educational level and age, confirming other studies with this population.

keywords

Psychodiagnostics. Psychological Assessment. Geriatrics. Learning. Dementia. 
ABREU, Izabella Dutra; FORLENZA, Orestes Vicente; BARROS, Hélio Lauar. Demência de Alzheimer: correlação entre memória e autonomia. Revista de Psiquiatria Clínica, São Paulo, v. 32, n. 3, p. 131-136, maio 2005.

AGRELL, Berit; DEHLIN, Ove. The Clock Drawing Test. Age and Ageing, Lund, v. 27, n. 3, p. 399-403, May 1998.

APRAHAMIAN, Ivan; DINIZ, Breno; IZBICKI, Rafael; RADANOVIC, Márcia; NUNES, Paula Villela; FORLENZA, Orestes Vicente. Optimizing the CAMCOG test in the screening for mild cognitive impairment and incipient dementia: saving time with relevant domains. International Journal of Geriatric Psychiatry, New York, v. 26, n. 4, p. 403-408, Apr. 2011.

APRAHAMIAN, Ivan; MARTINELLI, José Eduardo; CECATO, Juliana Francisca; IZBICKI, Rafael; YASSUDA, Monica Sanches. Can the CAMCOG be a good cognitive test for patients with Alzheimer's disease with low levels of education? International Psychogeriatrics, Cambridge, v. 23, n. 1, p. 96-101, Feb. 2011.

ARGIMON, Irani de Lima; TIMM, Luciana Almeida; RIGONI, Maisa Santos; OLIVEIRA, Margareth da Silva. Instrumentos de avaliação de memória em idosos: uma revisão. Revista Brasileira de Ciências do Envelhecimento Humano, Passo Fundo, v. 2, n. 2 p. 28-35, maio 2005 .

BATISTA, Natalia Cunha; CRISPIM, Natalia de Freitas. A interferência das relações familiares no processo de envelhecimento: Um enfoque no idoso hospitalizado. Revista Kairós Gerontologia, São Paulo, v. 15, n. 5, p. 169-189, set. 2012.

BENNETT, David Alan; WILSON, Richard Scott; SCHNEIDER, Julie et al. Natural history of mild cognitive impairment in older persons. Neurology, Chicago, v. 59, n. 2 , p. 198-205, July 2002.

BRITO-MASQUES, Paulo Roberto; CABRAL-FILHO, José Eulálio. Influence of age and schooling on the performance in a modified Mini-mental State Examination version: a study in Brazil northeast. Arquivos de Neuropsiquiatria, Recife, v. 63, n. 3A, 583-587, Mar. 2005

CAMPOS, Helton Rocha. Noções de psicometria. In: FUENTES, Daniel Dias; CAMARGO Candida Pires. Neuropsicologia: teoria e prática. Porto Alegre: Artmed, 2008. p. 89-102.

CARAMELLI, Paulo; BARBOSA, Maira Tonidandel. Como diagnosticar as quatro causas mais frequentes de demência? Revista Brasileira de Psiquiatria, São Paulo, v. 24, n. 1, p. 7-10, abr. 2002.

CECATO, Juliana Francisca; FIORESE, Bruna; MONTIEL, José Maria; BARTHOLOMEU, Daniel; MARTINELLI, José Eduardo. Clock Drawing Test in Elderly Individuals With Different Education Levels: Correlation With Clinical Dementia Rating. American Journal of Alzheimer's Disease and Other Dementias, Philadelphia, v. 27, n. 8, p. 620-624, Dec. 2012

CHARCHAT-FICHMAN, Helenice; CARAMELLI, Paulo; SAMESHIMA, Koichi; NITRINI, Ricardo. Declínio da capacidade cognitiva durante o envelhecimento. Revista Brasileira de Psiquiatria, São Paulo, v. 27, n. 12, p. 79-82, nov. 2005

CHARCHAT-FICHMAN, Helenice; NITRINI, Ricardo; CARAMELLI, Paulo; SAMESHIMA, Koichi. Investigação de marcadores clínicos dos estágios iniciais da doença de Alzheimer com testes neuropsicológicos computadorizados. Psicologia: Reflexão e Crítica, Rio Grande do Sul, v. 14, n. 2, p. 305-316, nov. 2001 
COOPER, David; EPKER, Melissa; LACRITZ, Laura. et al. Effects of practice on category fluency in Alzheimer's disease. American Academy of Clinical Neuropsychology, Dallas, v. 15, n. 1, p. 125-128, Feb. 2001

COOPER, David; LACRITZ, Laura; WEINER, Myron et al. Category fluency in mild cognitive impairment reduced effect of practice in test-retest conditions. Alzheimer disease and associated disorders, Houston, v. 18, n. 3, p. 120-122, July/Sep. 2004.

CORRÊA, Antonio Carlos Oliveira. Neuropsicologia da memória e sua avaliação. In: FUENTES, Daniel Dias; CAMARGO, Candida Pires. Neuropsicologia: teoria e prática. Porto Alegre: Artmed, 2008. p. 168-18.

DAMASCENO, Benito Pereira. Comprometimento cognitivo leve e doença de Alzheimer incipiente. In: CAIXETA, Leonardo. Demência: abordagem multidisciplinar. São Paulo: editora Atheneu, 2006. p. 129-140.

DINIZ, Breno Satler Oliveira; VOLPE, Fernando Madalena; TAVARES, Almir Ribeiro. Nível educacional e idade no desempenho do Miniexame do Estado Mental em idosos residentes na comunidade. Revista de Psiquiatria Clínica, São Paulo, v. 34, n. 1, p. 13-17, set. 2007.

EPEL, Elissa. Telomeres in a life-span perspective: a new Psychobiomarker? Current Directions in Psychological Science, San Francisco, v. 18, n. 1, p. 6-10, Feb. 2009.

EPEL, Elissa; BLACKBURN, Elizabeth; LIN, Jue; DHABHAR, Firdaus; ADLER, Nacy; MORROW, Jason; CAWTHON, Richard. Accelerated telomere shorting in response to life stress. Proceedings of the National Academy of Sciences of the United States of America, Boston, v. 101, n. 49, p. 17312-17315, Dec. 2008

FORMIGA, Francesc; SOTO, Alfons Lopes; SACANELLA, Emilio; JACOB, Ximenes; MASANÉS, Francisco; VIDAL, Marcelo. Valoración de la capacidad funcional después de un ingreso hospitalario en pacientes nonagenários. Medicina Clínica, Barcelona, v. 115, n. 18, p. 695-696, Abr. 2000.

GAINOTTI, Guido; MARRA, Carlo. Some aspects of memory disorders clearly distinguish dementia of the Alzheimer's type from depressive pseudo-dementia. Journal of clinical and experimental neuropsychology, Rome, v. 16, n. 1, p. 65-78, Feb. 1994.

GIOVANNETTI, Tania; LAMAR, Melissa et al. Different underlying mechanisms for deficits in concept formation in dementia. Archives of clinical neuropsychology, Oxford, v. 16, n. 6, p. 547-560, Aug. 2001

GOLD, Carl Artur; MARCHANT, Natalie Lia; KOUTSTAAL, Wilma et al. Conceptual fluency at test shifts recognition response bias in Alzheimer's disease: implications for increased false recognition. Neuropsychologia, Bethesda, v. 45, n. 12, p. 2791-2801, May 2007.

GOTTLIEB, Maria Grabriela Vale; SCHWANKE, Carla Helena Augustin; GOMES, Irenio; CRUZ, Ivana Beatrice Monica. Envelhecimento e Longevidade no Rio Grande do Sul: um perfil histórico, étnico e de morbi-mortalidade dos idosos. Revista Brasileira de Geriatria e Gerontologia, Rio de Janeiro, v. 14, n. 2, p. 365-380, jun. 2011.

HEINIK, Jeremia; SOLOMESH, Isaac; BLEICH, Avraham; BERKMAN, Paul. Are the Clock Drawing Test and the MMSE Combined Interchangeable with CAMCOG as a Dementia Evaluation Instrument in a Specialized Outpatient Setting? Journal of Geriatric Psychiatry and Neurology, Tel Aviv-Yafo, v. 16, n. 2, p. 74-79, June 2003.

LACERDA, Vandercy Soares; ZAMBOM, Melissa Pichi; CECATO, Juliana Francisca; BARTHOLOMEU, Daniel; MARTINELI, José Eduardo; MONTIEL, José Maria. Avaliação da Intervenção de atividades lúdicas em Idosos Institucionalizados. Encontro, Santo André, v. 16, n. 24, p. 47-63, dez. 2013.

LUDERS, Selenita Lia Alfonso; STORANI, Maria Silvia Barros. Demência: impacto para a família e a sociedade. In: PAPALÉO, Neto. Gerontologia. São Paulo: Editora Atheneu, 1996. p. 146-159. 
MARTINELLI, José Eduardo; CECATO, Juliana Francisca; BARTHOLOMEU, Daniel; MONTIEL, José Maria; MARTINELLI, Marcos de Oliveira. Comparison of Cognitive Functions between patients with thyroid dysfunction with and without astheno-emotional syndrome. E3 Journal of Medical Research, South Africa, v. 3, n. 2, p. 13-17, Feb. 2014.

MATALLANA, Diana; SANTACRUZ, Carolina; CANO, Carla et al. The relationship between education level and mini-mental state examination domains among older Mexican Americans. Journal of geriatric psychiatry and neurology, Bogota, v. 24, n. 1, p. 9-18, Mar. 2011.

MONTIEL, José Maria. Avaliação Neuropsicológica de pacientes com transtorno de Pânico. 2005. 306 f. Dissertação (Mestrado em Psicologia) - Universidade São Francisco, Itatiba. 2005.

MONTIEL, José Maria; CECATO, Juliana Francisca; BARTHOLOMEU, Daniel; MARTINELLI, José Eduardo. Evaluation of Montreal cognitive assessment for the differential diagnosis of mild cognitive impairment and Alzheimer-s disease in elderly patients with more than 5 years of schooling: Data from a Brazilian sample. Advances in Aging Research, Hong Kong, v. 2, n. 3, p. 121-129, Nov. 2013.

MORAES, Camila; PINTO, Jony Arrais; LOPES, Marcos Antonio; LITVOC, Julio; BOTTINO, Cassio Machado de Campos. Impact of sociodemographic and health variables on mini-mental state examination in a community-based sample o folder people. European Archives of Psychiatry and Clinical Neuroscience, Berlim, v. 260, n. 7 , p. 535-42, Oct. 2010.

NERI, Anita Liberalesso. Dependência e autonomia. In: NERI, Anita Liberalesso; GUARIENTO, Maria Elena (Orgs.). Assistência Ambulatorial ao Idoso. Campinas: Alínea, 2010. p. 31-44.

Contribuições da psicologia ao estudo e à intervenção no campo da velhice. Revista Brasileira de Ciências do Envelhecimento Humano, Passo Fundo, v. 1, n. 1, p. 69-80, abr. 2004.

Qualidade de vida na velhice e subjetividade. In: NERI, Anita Liberalesso (Org.). Qualidade de vida na velhice: enfoque multidisciplinar. Campinas: Editora Alínea, 2007. p. 13-62.

NITRINI, Ricardo; CARAMELLI, Paulo; BOTTINO, Cassio Machado de Campos; DAMASCENO, Benito Pereira; BRUCKI, Sonia Maria Dozzi; Anghina, Renato. Diagnóstico de doença de Alzheimer no Brasil: avaliação cognitiva e funcional. Arquivos de Neuropsiquiatria, São Paulo, v. 63, n. 3A, p. 720-727, abr. 2005.

NUNES, Paula Villela; DINIZ, Breno Satler Oliveira; RADANOVIC, Marcia et al. Camcog as a screening tool for diagnosis of mild cognitive impairment and dementia in a Brazilian clinical sample of moderate to high education. International journal of geriatric psychiatry, Glasgow, v. 23, n. 11, p. 1127-1133, Nov. 2008.

ORGANIZAÇÃO MUNDIAL DA SAÚDE. Classificação de transtornos mentais e de comportamento da CID-10. Porto Alegre: Artes Médicas, 1992.

RIBEIRO, Cristina Correa; YASSUDA, Monica Sanches. Cognição, estilo de vida e qualidade de vida na velhice. In: NERI, Anita Liberalesso. (Org.). Qualidade de vida na velhice: enfoque multidisciplinar. Campinas: Alínea, 2007. p. 189-204.

SANTOS, Ariene Angelini dos; PAVARINI, Sofia Cristina lost. Funcionalidade familiar de idoso com alterações cognitivas e diferentes contextos de vulnerabilidade social. Revista Eletrônica e Enfermagem, Goiânia, v. 13, n. 2, p. 361-367, abr. 2011.

SILVA, David; DAMASCENO, Benito Pereira. Demência na População de Pacientes do Hospital das Clínicas da Unicamp. Arquivos de Neuropsiquiatria, São Paulo, v. 60, n. 4, p. 996-999, dez. 2002. 
TEIXEIRA, Antonio Lucio; CARAMELLI, Paulo. Neuropsicologia das demências. In: FUENTES, Daniel Diaz; CAMARGO, Candida H. Pires. Neuropsicologia: teoria e prática. Porto Alegre: Artmed, 2008. p. 356-363.

TORRES, Gilson de Vasconcelos; REIS, Luciana Araújo dos; REIS, Luana Araujo dos; FERNANDES, Marcos Henrique. Qualidade de vida e fatores associados em idosos dependentes em uma cidade do interior do Nordeste. Jornal Brasileiro de Psiquiatria, São Paulo, v. 58, n. 1, p. 39-44, dez. 2009.

UNVERZAQT, Frederick; OGUNNIYI, Adesola; TALER, Vercic et al. Incidence and risk factors for cognitive impairment no dementia and mild cognitive impairment in African Americans. Alzheimer Disease and Associated Disorders, Indianapolis, v. 25, n. 1, p. 4-10, Jan./Mar. 2011

VALLS-PEDRET, Cinta; OLIVES, Juan; BOSCH, Barrera et al. Landscape test for assessing visual memory in Alzheimer's disease. Revista de Neurologia, Barcelona, v. 53, n. 1, p. 1-7, Jul. 2011

WANG, Hui; KARP, Anita; HERLITZ, Anna et al. Personality and lifestyles in relation to dementia incidence. Neurology, Stockholm, v. 72, n. 3, p. 253-59, Jan. 2009.

YASSUDA, Monica Sanches. Memória e envelhecimento saudável. In: FREITAS, Elizabete Viana; PY, Ligia. Tratado de Geriatria e Gerontologia. Rio de Janeiro: Editora Guanabara Koogan, 2002. p. 914-920.

YASSUDA, Monica Sanches; BATISTONI, Samila Sathler Tavares; FORTES, Andrea Garofe; NERI, Anita Liberalesso. Treino de memória no idoso saudável: benefícios e mecanismos. Psicologia: reflexão e crítica, São Paulo, v. 19, n. 3, p. 470-481, jan. 2006.

Recebido em: 16/04/2013

Aceite final em: 04/11/2014 